\title{
Gender-specific Associations of the Brain-derived Neurotrophic Factor Val66Met Polymorphism with Neurocognitive and Clinical Features in Schizophrenia
}

\author{
Sung-Wan Kim, Ju-Yeon Lee, Hee-Ju Kang, Seon-Young Kim, Kyung-Yeol Bae, Jae-Min Kim, II-Seon Shin, \\ Jin-Sang Yoon \\ Department of Psychiatry, Chonnam National University Medical School, Gwangju, Korea
}

\begin{abstract}
Objective: To explore associations of the brain-derived neurotrophic factor (BDNF) Val66Met polymorphism with cognitive functioning and psychopathology in patients with schizophrenia.

Methods: We included 133 subjects meeting the DSM-IV criteria for schizophrenia who were in the post-acute stage of the disease. BDNF Val66Met genotypes were identified via polymerase chain reaction. The computerized neurocognitive function battery, Positive and Negative Syndrome Scale (PANSS), Calgary Depression Scale for Schizophrenia (CDSS), Social and Occupational Functioning Scale (SOFAS), and the Subjective Well-being under Neuroleptic Treatment (SWN-K) were administered. Gender-stratified sub-analysis was also conducted to identify gender-specific patterns in the findings.

Results: In male patients, no significant difference in any measure by BDNF genotype was evident. In female patients, scores on the CDSS and total PANSS and all subscales were significantly higher in valine (Val) carriers. In addition, scores on the SOFAS and SWN-K were significantly lower in Val carriers. In terms of neurocognitive measures, female patients with the Val allele had significantly poorer reaction times and fewer correct responses on the Continuous Performance Test (CPT) and the Trail Making Test (Parts A and B). After adjustment of PANSS total scores and log-transformed CDSS scores, CPT outcomes were significantly poorer in female patients with than in those without the Val allele.

Conclusion: Gender-specific associations of the Val allele with poor neurocognitive function and more severe psychopathology were evident. Further studies are required to explore the mechanisms of these differences and the potential utility of the BDNF genotype as a predictor of outcome in patients with schizophrenia.
\end{abstract}

KEY WORDS: Brain-derived neurotrophic factor; Cognition; Gender; Genetic polymorphism; Schizophrenia; Val66Met.

\section{INTRODUCTION}

Neurocognitive impairment is a core feature of schizophrenia. Cognition is one of key domains of psychopathology that require dimensional assessment in the fifth edition of the Diagnostic and Statistical Manual of Mental Disorders (DSM-5). ${ }^{1)}$ Brain-derived neurotrophic factor (BDNF) has been widely investigated in the context of the neurodevelopmental hypothesis of schizophrenia, given the role played by the factor in development and maintenance of the central nervous system. ${ }^{2)}$ BDNF is a peptide involved in neuronal development, differentiation, and plasticity. In addition, BDNF modulates the expression

\footnotetext{
Received: December 3, 2015/Revised: January 1, 2016

Accepted: January 6, 2016

Address for correspondence: Jin-Sang Yoon, MD, PhD

Department of Psychiatry, Chonnam National University Medical

School, 160 Baekseo-ro, Dong-gu, Gwangju 61469, Korea

Tel: +82-62-220-6142, Fax: +82-62-225-2351

E-mail: jsyoon@chonnam.ac.kr
}

levels of dopamine D3 receptors ${ }^{3)}$ and is involved in the maturation and plasticity of the mesolimbic dopaminergic system. ${ }^{4,5)}$ A functional single-nucleotide polymorphism that creates a valine (Val)-to-methionine (Met) substitution at BDNF codon 66 (Val66Met) triggers aberrant sorting and release of mature BDNF via the activity-dependent secretion pathway. The Met variant of the Val66Met polymorphism is related to impaired BDNF release. ${ }^{6)}$ The effects of the BDNF Val66Met polymorphism on cognition have been extensively documented in healthy carriers. The Met allele has been reported to negatively affect hippocampal volume ${ }^{7)}$ and to alter neurocognitive functioning ${ }^{8)}$ in healthy subjects.

Although the Val66Met polymorphism per se may not be associated with a major risk of schizophrenia development, increasing evidence indicates that the polymorphism modulates several clinical features of the illness, as well as neurocognitive functioning. ${ }^{2)}$ Many previous studies have shown that the 66Met allele is asso-

() This is an Open-Access article distributed under the terms of the Creative Commons Attribution Non-Commercial License (http://creativecommons.org/licenses/by-nc/4.0) which permits unrestricted non-commercial use, distribution, and reproduction in any medium, provided the original work is properly cited 
ciated with impairments in episodic memory, executive function, attentional processing, visuospatial ability, and cognitive flexibility in patients with schizophrenia. However, one earlier work found that the Val allele was associated with reduced memory function in patients with psychosis, but not in a healthy population. ${ }^{9)}$ A recent meta-analysis found that patients with schizophrenia differing in BDNF genotype did not exhibit significant between-genotype differences in most neurocognitive domains. In addition, any correlations between peripheral BDNF levels and neurocognitive phenotypes were minimal. ${ }^{10)}$

Such inconsistent results require further evaluation. One possible explanation is that some effects of the BDNF polymorphism are gender specific; a few prior studies found that BDNF levels differed by gender. ${ }^{11,12)}$ In addition, a few recent studies have identified between-gender differences in associations of BDNF levels and the Val66Met polymorphism with cognitive impairment in patients with schizophrenia. ${ }^{13,14)}$ The sex hormones are well known to influence BDNF levels. ${ }^{15)}$ Therefore, in the present study, we explored the association between the BDNF Val66Met polymorphism and cognitive function in patients with schizophrenia, paying particular attention to possible between-gender differences, which were explored via gender-stratified analysis.

\section{METHODS}

\section{Subjects}

Subjects meeting the criteria of the fourth edition of DSM (DSM-IV) ${ }^{16)}$ for schizophrenia were enrolled. All patients were required to be symptomatically stable, as judged by their treating psychiatrists, to allow evaluation of neurocognitive functioning, and to have experienced no change in medication for a minimum of 2 weeks prior to enrollment. The key exclusion criteria were acute psychotic symptoms including severe behavioral disturbance, severe and unstable physical illnesses, and a diagnosis of mental retardation. The studies were carried out according to the latest version of the Declaration of Helsinki and were approved by the Chonnam National University Hospital Institutional Review Board. All patients gave written informed consent before participating in the study.

\section{Psychiatric Measures}

The sociodemographic and clinical characteristics recorded included age, gender, duration of illness, type of antipsychotic (risperidone or other) prescribed, and ris- peridone-equivalent dosages, which was calculated from daily defined dose. ${ }^{17)}$ Psychiatric measures included the Positive and Negative Syndrome Scale (PANSS) ${ }^{18,19)}$ to assess psychotic symptoms and other psychopathologies; the Social and Occupational Functioning Scale (SOFAS). ${ }^{16)}$ to evaluate general functioning; the Calgary Depression Scale for Schizophrenia (CDSS) ${ }^{20,21)}$ to measure depressive symptoms in patients with schizophrenia; ${ }^{22)}$ and the Subjective Well-being under Neuroleptic Treatment-short form $(\mathrm{SWN}-\mathrm{K})^{23,24)}$ to assess quality of life in patients with schizophrenia. Higher scores of the PANSS and the CDSS represents more severe psychopathology, whereas higher scores of the SOFAS and the SWN-K represents better social functioning and quality of life, respectively.

\section{Neurocognitive Assessment}

A computerized battery of neurocognitive tests, standardized for the Korean population, ${ }^{25)}$ was administered to all participants. The neurocognitive tests used in the study were as follows: Attention span, vigilance, and working memory were measured by forward and backward Digit Span Test. Verbal memory was assessed by the modified Rey Auditory Verbal Learning Test. ${ }^{26,27)}$ Long-term delayed recall memory after 20 minutes was used as an outcome measure of verbal learning test. Executive function and cognitive flexibility were measured by numbers of categories completed on the Wisconsin Card Sorting Test. ${ }^{28)}$ Sustained attention was assessed by the number of correct responses and reaction time on the Continuous Performance Test (CPT). Finally, the Trail Making Test $(\mathrm{TMT})^{29)}$ was performed; Part A of the test measures visuomotor speed and attention and Part B measures executive function and visuospatial working memory.

\section{Genotype}

Venous blood samples were obtained from all participants, and DNA was extracted using standard procedures. Polymerase chain reaction (PCR) and PCR-based restriction fragment length polymorphism assays were performed. Hydrolysis by the restriction enzyme BbrPI identified PCR products characteristic of the 66Val (490and 208-bp fragments) and the 66Met (a 698-bp fragment) alleles; the genotypes were categorized as Val/Val, Val/Met, or Met/Met.

\section{Statistical Analysis}

Deviation from Hardy-Weinberg equilibrium was assessed using the chi-squared test. Subjects were divided into two groups by Val allele status: Val/Val and Val/Met 
vs. Met/Met. Sociodemographic and clinical variables and neurocognitive outcome measures were compared according to Val allele status and gender, respectively using the chi-squared test, the independent $t$-test, and the Mann-Whitney $U$-test, as appropriate. Variables and outcome measures were compared by Val allele status by gender using same analyses. The effects of genotype on outcome measures and their interactions with gender were assessed using general linear models. Same exploratory analysis on neurocognitive function was conducted in subjects who received risperidone monotherapy to control confounding effects of antipsychotic medication on neurocognitive function. Finally, we conducted an analysis of covariance (ANCOVA) to compare the effects of the Val allele on cognitive functioning after adjustment for variables that significantly differed by BDNF genotype. Neurocognitive function measures that were statistically significant in unadjusted analysis were used as dependent variables, with Val allele carrier as the categorical independent variable in ANCOVA. Values that were not normally distributed were entered as covariates after log transformation. All statistical tests were two tailed, and the significance level was set at $p<0.05$. All analyses were performed with IBM SPSS Statistics software version 21.0 (IBM Co., Armonk, NY, USA).

\section{RESULTS}

\section{Subjects}

A total of 133 subjects, 71 females (53.4\%) and 62 males $(46.6 \%)$, participated in the study. The mean participant age was 33.6 years (standard deviation [SD], 8.7 years), and the median number of years of education was 14 (interquartile range [IQR], 12-16 years). The median duration of illness was 5.6 years (IQR, 2.6-10.2 years), and about $75 \%$ of all participants received risperidone monotherapy.

\section{Sociodemographic and Clinical Characteristics and Neurocognitive Functioning by BDNF Polymorphism}

Of all patients, $23.3 \%$ were homozygous for the Met allele, consistent with the existence of Hardy-Weinberg equilibrium $(p>0.05)$. No significant difference in sociodemographic characteristics by Val allele status was evident (Table 1). In terms of psychiatric measures, the total PANSS and negative symptom subscale scores were significantly higher in patients with Val allele than in those with the Met/Met genotype. The CDSS, SOFAS and SWN scores did not significantly differ by Val allele.
Performances on the TMT Part B and correct response of the CPT were significantly poorer in patients with Val allele than in those without Val allele. No sociodemographic or clinical characteristic, and no measure of neurocognitive functioning, differed by gender.

\section{Sociodemographic and Clinical Characteristics and Neurocognitive Functioning by Val Allele Status in Each Gender}

Table 2 compares sociodemographic and clinical characteristics and neurocognitive measures by Val allele status by gender. In males, no significant difference was observed based on Val allele status. However, in females, the CDSS and total PANSS and all subscale scores, were significantly higher in Val carriers, and the SOFAS and SWN scores significantly lower. In terms of neurocognitive measures, females with the Val allele had significantly longer response times on the CPT, TMT-Part A, and TMT-Part B and significantly fewer correct responses on the CPT compared with those without the Val allele. Significant gender $\times$ gene interactions were evident for all psychiatric scales and the two CPT outcomes (reaction time and correct response). Exploratory analysis in risperidone monotherapy group showed almost same results; females with the Val allele had longer response times on the CPT, TMT-Part A, and TMT-Part B ( $p=0.046,0.055$, and 0.026 , respectively) and significantly fewer correct responses on the CPT $(p=0.028)$ compared with those without the Val allele (data not shown).

Table 3 shows the results of multivariate analysis of neurocognitive measures according to Val allele status in female patients. After adjustment for the PANSS total score and the log-transformed CDSS score as covariates, the reaction time was significantly longer, and correct responses significantly fewer in females with than in those without a Val allele.

\section{DISCUSSION}

The principal findings of the present study were that female Val allele carriers with schizophrenia exhibited significantly more severe psychotic and depressive symptoms, poorer social functioning, and a lower quality of life than did homozygous Met/Met patients. In addition, Val allele carriers scored lower on tests of neurocognitive functioning than did others. After adjustment for psychotic and depressive symptoms, significant betweengroup differences remained in terms of sustained attention and measures of psychomotor speed. We observed sig- 


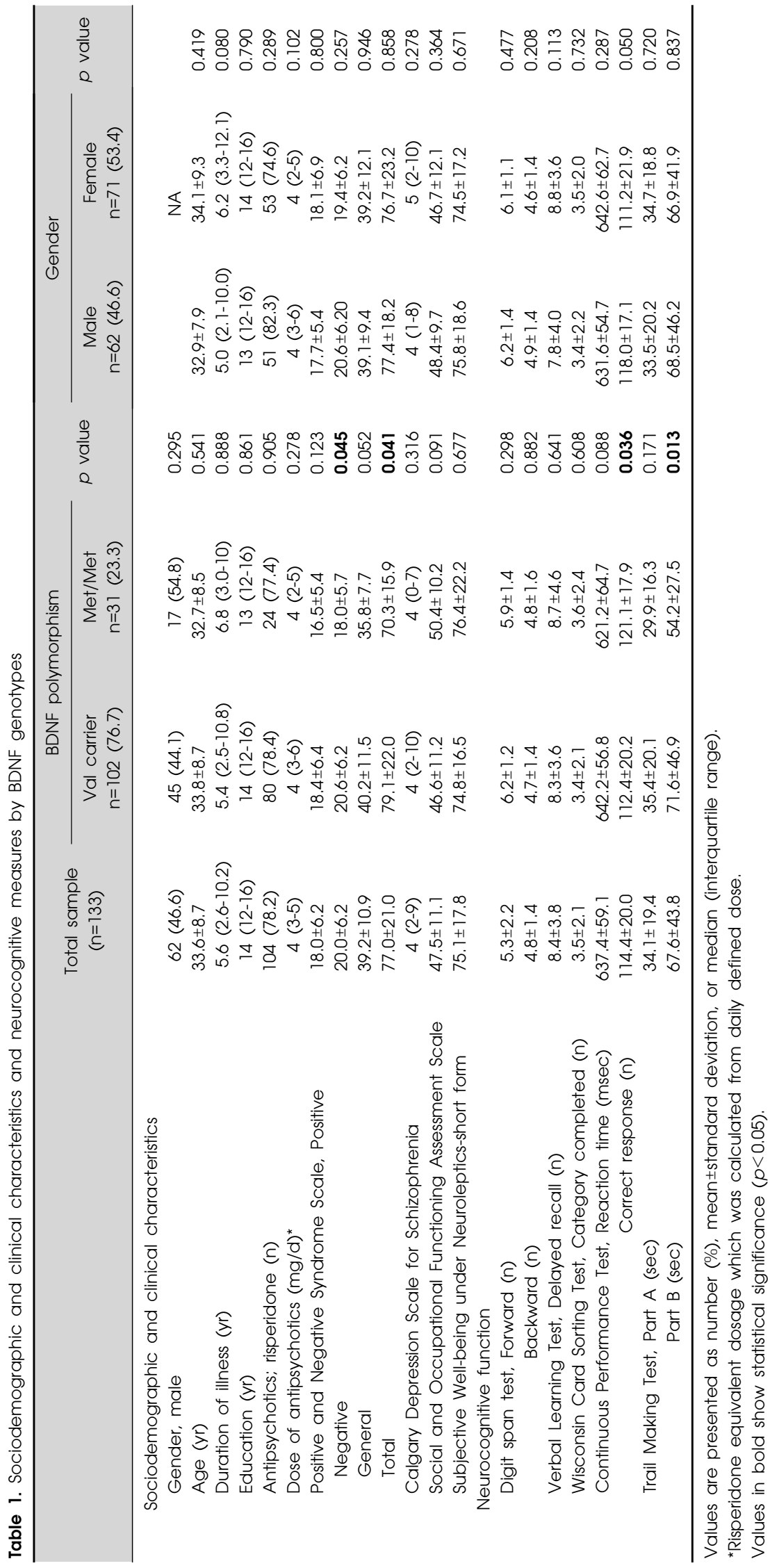




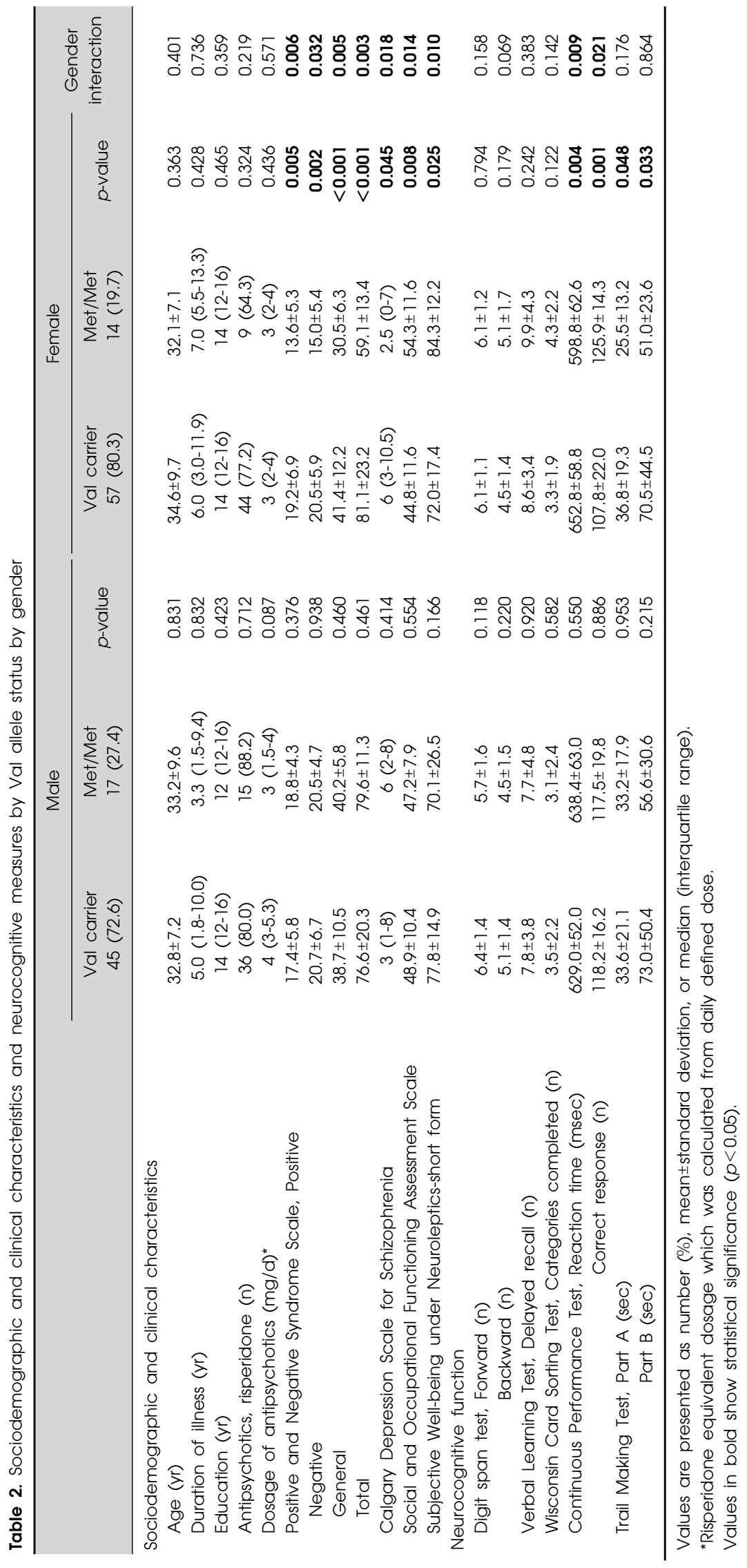


Table 3. Multivariate analysis of neurocognitive measures according to Val allele status in female patients

\begin{tabular}{|c|c|c|c|}
\hline & Val carrier $n=76(57.1)$ & Met/Met $n=31$ (23.3) & $p$ value \\
\hline Continuous Performance Test, Reaction time (msec) & $652(636-668)$ & $602(566-637)$ & 0.015 \\
\hline Correct response $(n)$ & $108.0(102.4-113.7)$ & $125.0(112.6-137.5)$ & 0.018 \\
\hline Trail Making Test, Part A (sec) & $36.4(31.5-41.3)$ & $27.2(16.3-38.1)$ & 0.137 \\
\hline Part B (sec) & $69.7(58.5-80.9)$ & $54.7(29.9-79.5)$ & 0.287 \\
\hline
\end{tabular}

Values are presented as adjusted mean ( $95 \%$ confidence interval).

Values in bold show statistical significance $(p<0.05)$.

Adjusted for Positive And Negative Syndrome Scale total score and log-transformed Calgary Depression Scale for Schizophrenia score.

nificant gender interactions when the effects of the BDNF polymorphism on neurocognitive functioning and psychopathology were explored in patients with schizophrenia.

Our present findings are not compatible with the notion that BDNF is involved in neurogenesis and neuroprotection; the Val allele is known to increase secretion of BDNF. ${ }^{6)}$ Most studies on healthy populations have found that the BDNF Met allele was associated with poor neurocognitive functioning; ${ }^{7,8)}$ many studies with schizophrenia patients have yielded similar results. ${ }^{2}$ However, a recent meta-analysis found no evidence that the BDNF Val66Met polymorphism was associated with neurocognitive functioning in patients with schizophrenia. ${ }^{10)}$ Moreover, we found, in the present study, that the Val allele was associated with poor neurocognitive functioning only in female patients. We offer two possible explanations. First, the Val66Met allele may affect cognitive functioning of patients with schizophrenia in a manner different from the effect of the allele on healthy subjects. Several studies have found that the effects of the BDNF Val66Met polymorphism differed between healthy populations and patients with schizophrenia, suggestive of illness-related modulation of the effect of the BDNF Val66Met allele. ${ }^{9,30)}$ In particular, regulation of glutamate and dopamine release by BDNF may be associated with illness specific findings of previous studies in patients with schizophrenia. ${ }^{31,32)}$ Second, the fact that the effects of the BDNF Val66Met polymorphism observed in our present study are gender specific may explain why some earlier data were inconsistent. Indeed, a few previous studies found that the association of the Met allele with poor neurocognitive function in patients with schizophrenia was confined to males. ${ }^{13,33)}$ A recent animal study suggested that BDNF-tropomyosin-related kinase B (TrkB) signaling may be controlled in a gender-specific manner. ${ }^{34)}$ In addition, one previous study found that patients with Met alleles performed significantly better executive function task than did those without Met alleles in female patients with Parkinson's disease, as we also found. ${ }^{35)}$
The gender-specific influence of the BDNF Val66Met polymorphism may be explained by the fact that BDNF and sexual hormones interact significantly. Animal studies suggest that estrogen increases BDNF levels. ${ }^{36-38)}$ Thus, female patients with schizophrenia may express more BDNF than males ones do, ${ }^{14)}$ and this may be further exacerbated in patients with higher BDNF secretion rates (Val carriers). BDNF controls the development of dopamine D3 receptors and maintenance of receptor expression. Activation of these receptors inhibited working memory ability in animal experiments using D3 receptor agonists and antagonists. ${ }^{3)}$ Therefore, relatively elevated secretion of BDNF (in Val carriers) may negatively impact neurocognitive functioning by increasing D3 receptor expression. Indeed, a study on patients with Parkinson's disease, in whom the dopaminergic system is compromised, showed that those with the Met allele performed significantly better on working memory tasks and delayed recall tests. ${ }^{35,39)}$ We also speculate that sex hormones such as estrogen may play roles in the sexually dimorphic effects of BDNF genotypes.

In the present study, the Val allele was significantly associated with more severe psychotic and depressive symptoms. This seems to contradict previous findings showing negative relationships between BDNF levels and the severity of depression in populations with depressive disorder. ${ }^{40-42)}$ Moreover, the Met allele was associated with a higher depression score in patients with schizophrenia. $^{43)}$ However, a positive correlation between BDNF level and the severity of depression has been reported in females, but not males. ${ }^{44)}$ In addition, the Val allele was associated with an elevated cortisol response to stress and increased anxiety and neurotisim. ${ }^{45-48)}$ Further study is warranted to investigate the roles played by BDNF polymorphisms and BDNF levels in depressed patients with schizophrenia.

Associations between the Val66Met polymorphism and the severity of psychotic symptoms in patients with schizophrenia have been inconsistent. In one study on Chinese 
inpatients, the Val allele was associated with lower PANSS scores, particularly on the negative and depression panels. ${ }^{43)}$ In addition, in a study using CAITIE data, the Met allele was associated with treatment resistance. ${ }^{49}$ By contrast, a study on Polish patients with paranoid schizophrenia found that the Val/Val genotype was associated with more severe symptoms in males but not in females. ${ }^{50)}$ In a previous study on Chinese patients with schizophrenia, the Val allele tended to be associated with higher subscores on negative symptoms of the PANSS, but no gender-stratified sub-analysis was performed. ${ }^{51}$ The BDNF Met allele was found to be associated with poor BDNF secretion, reduced expression of dopamine D3 receptor, and less severe psychotic symptoms. ${ }^{52)}$ In addition, as previously suggested, it may be the case that the Val genotype is associated with treatment-resistant symptoms, resulting in reports of more severe symptoms. ${ }^{53)}$ This would explain why the Val allele was associated with more severe psychotic or more treatment-resistant symptoms both in earlier studies and in the present work.

A few limitations of our work should be borne in mind. First, BDNF genotypes are trait dependent, whereas assessments of cognitive functioning and the intensity of illness using the PANSS are state dependent. We believe that the post-acute status of our present patients and their relatively low PANSS scores reduce the confounding problem. However, relationships between clinical symptoms and genotypes warrant further investigation in first-episode drug-naïve patients. ${ }^{51)}$ Second, gene $\times$ gene and gene $\times$ environment interactions, as well as epigenetic modification, may influence the role played by the BDNF Val66Met polymorphism in patients with schizophrenia. Third, it remains possible that the effects of the Val66Met polymorphism on the cognition and psychopathology of females with schizophrenia may be attributable to linkage disequilibrium between this polymorphism and a functional polymorphism lying in or near the BDNF gene. ${ }^{54)}$ Fourth, all of our patients were Korean. Indeed, there is some evidence that Val66Met genotype frequencies may vary by ethnicity, with the Met allele being more prevalent in Asians than in other ethnic groups. ${ }^{55)}$ Finally, sample size was relatively small to have enough statistical power, particularly for interaction effects of gender.

In conclusion, the present study found out that gender-specific associations of the Val allele with poor neurocognitive function and more severe psychopathology. Furthermore, the significance of the association between the Val allele and poor neurocognitive functioning was maintained after adjustment for severity of psychopathol- ogy in female patients with schizophrenia. These results suggest that BDNF action may vary by gender in patients with schizophrenia, explaining the conflicting results of earlier genetic studies. Further studies are required to explore the mechanisms of these differences and the potential utility of the BDNF genotype as a predictor of outcome in patients with schizophrenia. Large samples of subjects varying in ethnicity should be studied longitudinally.

\section{Acknowledgments}

This research was supported by Basic Science Research Program through the National Research Foundation of Korea (NRF) funded by the Ministry of Education (NRF-2014R1A1A4A01005245).

S.W.K. has received honoraria from Janssen and Otsuka. J.S.Y. has received unrestricted research funds from Sanofi-Aventis, Janssen, Roche, Otsuka, and Lundbeck. Other authors have declared that there are no conflicts of interest in relation to the subject of this study.

\section{REFERENCES}

1. American Psychiatric Association. Diagnostic and statistical manual of mental disorders. 5th ed. Arlington, VA:American Psychiatric Publishing;2013.

2. Notaras M, Hill R, van den Buuse M. A role for the BDNF gene Val66Met polymorphism in schizophrenia? A comprehensive review. Neurosci Biobehav Rev 2015;51:15-30.

3. Guillin O, Diaz J, Carroll P, Griffon N, Schwartz JC, Sokoloff P. BDNF controls dopamine D3 receptor expression and triggers behavioural sensitization. Nature 2001;411:86-89.

4. Baker SA, Stanford LE, Brown RE, Hagg T. Maturation but not survival of dopaminergic nigrostriatal neurons is affected in developing and aging BDNF-deficient mice. Brain Res 2005;1039:177-188.

5. Guillin O, Griffon N, Diaz J, Le Foll B, Bezard E, Gross $\mathrm{C}$, et al. Brain-derived neurotrophic factor and the plasticity of the mesolimbic dopamine pathway. Int Rev Neurobiol 2004;59:425-444.

6. Egan MF, Kojima M, Callicott JH, Goldberg TE, Kolachana $\mathrm{BS}$, Bertolino A, et al. The BDNF val66met polymorphism affects activity-dependent secretion of BDNF and human memory and hippocampal function. Cell 2003;112:257-269.

7. Bueller JA, Aftab M, Sen S, Gomez-Hassan D, Burmeister M, Zubieta JK. BDNF Val66Met allele is associated with reduced hippocampal volume in healthy subjects. Biol Psychiatry 2006;59:812-815.

8. Dincheva I, Glatt CE, Lee FS. Impact of the BDNF Val66Met polymorphism on cognition: implications for behavioral genetics. Neuroscientist 2012;18:439-451.

9. Smith GN, Thornton AE, Lang DJ, Macewan GW, Ehmann TS, Kopala LC, et al. Hippocampal volume and the brain-derived neurotrophic factor Val66Met polymorphism in first episode psychosis. Schizophr Res 2012;134:253-259.

10. Ahmed AO, Mantini AM, Fridberg DJ, Buckley PF. Brain-derived neurotrophic factor (BDNF) and neurocognitive deficits in people with schizophrenia: a meta- 
analysis. Psychiatry Res 2015;226:1-13.

11. Xiu MH, Hui L, Dang YF, Hou TD, Zhang CX, Zheng YL, et al. Decreased serum BDNF levels in chronic institutionalized schizophrenia on long-term treatment with typical and atypical antipsychotics. Prog Neuropsychopharmacol Biol Psychiatry 2009;33:1508-1512.

12. Pillai A, Bruno D, Sarreal AS, Hernando RT, Saint-Louis LA, Nierenberg J, et al. Plasma BDNF levels vary in relation to body weight in females. PLoS One 2012;7:e39358.

13. Lu W, Zhang C, Yi Z, Li Z, Wu Z, Fang Y. Association between BDNF Val66Met polymorphism and cognitive performance in antipsychotic-naïve patients with schizophrenia. J Mol Neurosci 2012;47:505-510.

14. Zhang XY, Chen DC, Tan YL, Tan SP, Wang ZR, Yang $\mathrm{FD}$, et al. Gender difference in association of cognition with $B D N F$ in chronic schizophrenia. Psychoneuroendocrinology 2014;48:136-146.

15. Sohrabji F, Lewis DK. Estrogen-BDNF interactions: implications for neurodegenerative diseases. Front Neuroendocrinol 2006;27:404-414.

16. American Psychiatric Association. Diagnostic and statistical manual of mental disorders. 4th ed. Washington, DC: American Psychiatric Press Inc.;1994.

17. Patel MX, Arista IA, Taylor M, Barnes TR. How to compare doses of different antipsychotics: a systematic review of methods. Schizophr Res 2013;149:141-148.

18. Kay SR, Fiszbein A, Opler LA. The positive and negative syndrome scale (PANSS) for schizophrenia. Schizophr Bull 1987;13:261-276.

19. Yi JS, Ahn YM, Shin HK, An SK, Joo YH, Kim SH, et al. Reliability and validity of the Korean version of the positive and negative syndrome scale. $J$ Korean Neuropsychiatr Assoc 2001;40:1090-1105.

20. Addington D, Addington J, Schissel B. A depression rating scale for schizophrenics. Schizophr Res 1990;3:247-251.

21. Kim YK, Won SD, Lee KM, Choi HS, Jang HS, Lee BH, et al. A study on the reliability and validity of the Korean version of the Calgary Depression Scale for Schizophrenia (K-CDSS). J Korean Neuropsychiatr Assoc 2005;44:446-455.

22. Kim SW, Kim SJ, Yoon BH, Kim JM, Shin IS, Hwang MY, et al. Diagnostic validity of assessment scales for depression in patients with schizophrenia. Psychiatry Res 2006;144: 57-63.

23. Naber D, Moritz S, Lambert M, Pajonk FG, Holzbach R, Mass $\mathrm{R}$, et al. Improvement of schizophrenic patients subjective well-being under atypical antipsychotic drugs. Schizophr Res 2001;50:79-88.

24. Kim SW, Shin IS, Kim JM, Yoo JA, Ahn YM, Kwon JS, et al. A validation study of the Korean version of the subjective well-being under neuroleptic treatment scale-short form. Korean J Psychopharmacol 2007;18:221-230.

25. Ha KS, Kwon JS, Lyoo IK, Kong SW, Lee DW, Youn T. Development and standardization process, and factor analysis of the computerized cognitive function test system for Korea adults. J Korean Neuropsychiatr Assoc 2002;41:551-562.

26. Rey A. L'examen clinique en psychologie. Paris:Presses Universitaires de France; 1964.

27. Kwon JS, Lyoo IK, Hong KS, Yeon BK, Ha KS. Development and standardization of the computerized memory assessment for Korean adults. J Korean Neuropsychiatr Assoc 2002;41:347-362.

28. Heaton RK, Chelune GJ, Talley JL, Kay GG, Curtiss G. Wisconsin Card Sorting Test Manual. Odessa, FL:Psychological Assessment Resources;1993.
29. Lezak MD. Neuropsychological assessment. 3rd ed. New York:Oxford University Press; 1995.

30. Dempster E, Toulopoulou T, McDonald C, Bramon E, Walshe M, Filbey F, et al. Association between BDNF val66 met genotype and episodic memory. Am J Med Genet B Neuropsychiatr Genet 2005;134B:73-75.

31. Jovanovic JN, Czernik AJ, Fienberg AA, Greengard P, Sihra TS. Synapsins as mediators of BDNF-enhanced neurotransmitter release. Nat Neurosci 2000;3:323-329.

32. Goggi J, Pullar IA, Carney SL, Bradford HF. Signalling pathways involved in the short-term potentiation of dopamine release by BDNF. Brain Res 2003;968:156-161.

33. Chung S, Chung HY, Jung J, Chang JK, Hong JP. Association among aggressiveness, neurocognitive function, and the Val66Met polymorphism of brain-derived neurotrophic factor gene in male schizophrenic patients. Compr Psychiatry 2010;51:367-372.

34. Hill RA, van den Buuse M. Sex-dependent and regionspecific changes in TrkB signaling in BDNF heterozygous mice. Brain Res 2011;1384:51-60.

35. Foltynie T, Lewis SG, Goldberg TE, Blackwell AD, Kolachana BS, Weinberger DR, et al. The BDNF Val66Met polymorphism has a gender specific influence on planning ability in Parkinson's disease. J Neurol 2005;252:833-838.

36. Berchtold NC, Kesslak JP, Pike CJ, Adlard PA, Cotman $\mathrm{CW}$. Estrogen and exercise interact to regulate brain-derived neurotrophic factor $m R N A$ and protein expression in the hippocampus. Eur J Neurosci 2001;14:1992-2002.

37. Ivanova T, Küppers E, Engele J, Beyer C. Estrogen stimulates brain-derived neurotrophic factor expression in embryonic mouse midbrain neurons through a membranemediated and calcium-dependent mechanism. J Neurosci Res 2001;66:221-230.

38. Solum DT, Handa RJ. Estrogen regulates the development of brain-derived neurotrophic factor $m R N A$ and protein in the rat hippocampus. J Neurosci 2002;22:2650-2659.

39. Białecka M, Kurzawski M, Roszmann A, Robowski P, Sitek EJ, Honczarenko K, et al. BDNF G196A (Val66Met) polymorphism associated with cognitive impairment in Parkinson's disease. Neurosci Lett 2014;561:86-90.

40. Karege F, Perret G, Bondolfi G, Schwald M, Bertschy G, Aubry JM. Decreased serum brain-derived neurotrophic factor levels in major depressed patients. Psychiatry Res 2002;109:143-148.

41. Shimizu E, Hashimoto K, Okamura N, Koike K, Komatsu $\mathrm{N}$, Kumakiri C, et al. Alterations of serum levels of brain-derived neurotrophic factor (BDNF) in depressed patients with or without antidepressants. Biol Psychiatry 2003;54:70-75.

42. Dell'Osso L, Del Debbio A, Veltri A, Bianchi C, Roncaglia I, Carlini M, et al. Associations between brain-derived neurotrophic factor plasma levels and severity of the illness, recurrence and symptoms in depressed patients. Neuropsychobiology 2010;62:207-212.

43. Sun MM, Yang LM, Wang Y, Feng X, Cui KY, Liu LF, et al. BDNF Val66Met polymorphism and anxiety/ depression symptoms in schizophrenia in a Chinese Han population. Psychiatr Genet 2013;23:124-129.

44. Kreinin A, Lisson S, Nesher E, Schneider J, Bergman J, Farhat $\mathrm{K}$, et al. Blood BDNF level is gender specific in severe depression. PLoS One 2015;10:e0127643.

45. Shalev I, Lerer E, Israel S, Uzefovsky F, Gritsenko I, Mankuta D, et al. BDNF Val66Met polymorphism is associated with HPA axis reactivity to psychological stress characterized by genotype and gender interactions. Psy- 
choneuroendocrinology 2009;34:382-388.

46. Alexander N, Osinsky R, Schmitz A, Mueller E, Kuepper Y, Hennig J. The BDNF Val66Met polymorphism affects HPA-axis reactivity to acute stress. Psychoneuroendocrinology 2010;35:949-953.

47. Lang UE, Hellweg R, Kalus P, Bajbouj M, Lenzen KP, Sander T, et al. Association of a functional BDNF polymorphism and anxiety-related personality traits. Psychopharmacology (Berl) 2005;180:95-99.

48. Sen S, Nesse RM, Stoltenberg SF, Li S, Gleiberman L, Chakravarti $\mathrm{A}$, et al. A BDNF coding variant is associated with the NEO personality inventory domain neuroticism, a risk factor for depression. Neuropsychopharmacology 2003;28:397-401.

49. Zhang JP, Lencz T, Geisler S, DeRosse P, Bromet EJ, Malhotra AK. Genetic variation in BDNF is associated with antipsychotic treatment resistance in patients with schizophrenia. Schizophr Res 2013;146:285-288.

50. Suchanek R, Owczarek A, Paul-Samojedny M, Kowalczyk $\mathrm{M}$, Kucia K, Kowalski J. BDNF val66met polymorphism is associated with age at onset and intensity of symptoms of paranoid schizophrenia in a Polish population. $J$ Neuropsychiatry Clin Neurosci 2013;25:88-94.

51. Zhou DH, Yan QZ, Yan XM, Li CB, Fang H, Zheng YL, et al. The study of BDNF Val66Met polymorphism in Chinese schizophrenic patients. Prog Neuropsychopharmacol Biol Psychiatry 2010;34:930-933.

52. Hashim HM, Fawzy N, Fawzi MM, Karam RA. Brain-derived neurotrophic factor Val66Met polymorphism and obsessive-compulsive symptoms in Egyptian schizophrenia patients. J Psychiatr Res 2012;46:762-766.

53. Numata S, Ueno S, Iga J, Yamauchi K, Hongwei S, Ohta $\mathrm{K}$, et al. Brain-derived neurotrophic factor (BDNF) Val66Met polymorphism in schizophrenia is associated with age at onset and symptoms. Neurosci Lett 2006;401:1-5.

54. Zintzaras E. Brain-derived neurotrophic factor gene polymorphisms and schizophrenia: a meta-analysis. Psychiatr Genet 2007;17:69-75.

55. Kim JM, Stewart R, Kim SW, Yang SJ, Shin IS, Kim YH, et al. BDNF genotype potentially modifying the association between incident stroke and depression. Neurobiol Aging 2008;29:789-792. 